\title{
Cutaneous Pseudolymphoma Derived from Ruptured Milia
}

\author{
Minseok Lee, Jemin Kim, Do Young Kim \\ Department of Dermatology, Severance Hospital, Cutaneous Biology Research Institute, Yonsei University College of Medicine, Seoul, \\ Korea
}

\section{Dear Editor:}

Cutaneous pseudolymphoma (CPL) is an inflammatory condition described as a heterogeneous group of benign reactive $\mathrm{T}$-cell or B-cell lymphoproliferative processes of diverse causes ${ }^{1}$. CPL has been associated with variable causes such as drugs, trauma, infections, inflammatory dermatoses, and reaction to foreign antigens introduced by arthropod bites, stings, infestation, tattoos, and vaccinations ${ }^{2,3}$. Although most cases are idiopathic, these associations suggest that $\mathrm{CPL}$ may be a reactive response to newly encountered antigens ${ }^{4}$. Here, we report a case of recurrent $\mathrm{CPL}$ derived from the rupture of multiple milia on the face.

A 72-year old woman presented with multiple confluent erythematous plaques on the forehead and left side of her face of 6-years duration (Fig. 1A). The patient had a medical history of hypertension although both clinical examination and blood test findings were otherwise unremarkable. She had no other symptoms such as fever, or history of recent travel, localised trauma, medication intake, or insect bites. Histologic examination of punch biopsy specimens taken from the plaques on her forehead revealed lymphoid nodules consisting of dense mixed lymphocytic infiltrates (Fig. 2A). Infiltrating cells were predominantly mixed with CD20 expressing cells and CD3 expressing cells (Supplementary Fig. 1A, B). The cells were focally positive for BCL2 and BCL6; CD43 was expressed only by $\mathrm{T}$ lymphocytes (Supplementary Fig. 1C E). On the basis of the histological findings, a diagnosis of CPL was suggested. The patient was treated with oral prednisolone and topical steroids, and the lesions temporarily subsided. However, the lesions recurred, and upon closer examination, multiple milia were found within the lesions (Fig. 1B). Another biopsy was performed to capture the new lesions that included milia; histopathology showed dense lymphocytic infiltration recognizably derived from ruptured milia (Fig. 2B).

Based on the above findings, a new diagnosis of milium with pseudolymphoma was made. The lesions markedly subsided with oral prednisolone and intra-lesional triamcinolone injections. However, on subsequent follow-up, the lesions returned and were distributed around the site of recurrent milia. Thus, the milia were extracted, and the recurrence of CPL remarkably subsided. Subsequently, the symptoms were well-controlled with oral cyclosporine and intra-lesional injections of triamcinolone (Fig. 1C).

CPL has a wide range of presentations and histological features that may be misinterpreted clinically and histologically as cutaneous lymphomas.

In our case, the lesions were distributed within areas of milia; the histopathological findings of lymphocytic infiltrations along the ruptured milia suggested that $\mathrm{CPL}$ arose from the ruptured milia. In addition, by treating the milia, the CPL lesions improved and the recurrences were markedly reduced, which further indicated their association.

It was reported that persistent stimulation caused by nodular scabies, arthropod bites, and retained foreign materials can promote chronic antigenic reactions ${ }^{5}$. Similarly, keratin protein from the ruptured milia may act as an antigen that causes a persistent reaction, subsequently leading

Received September 15, 2017, Accepted for publication December 14, 2017

Corresponding author: Do Young Kim, Department of Dermatology, Severance Hospital, Cutaneous Biology Research Institute, Yonsei University College of Medicine, 50-1 Yonsei-ro, Seodaemun-gu, Seoul 03722, Korea. Tel: 82-2-2228-2080, Fax: 82-2-393-9157, E-mail: dykim@yuhs.ac ORCID: https://orcid.org/0000-0002-0194-9854

This is an Open Access article distributed under the terms of the Creative Commons Attribution Non-Commercial License (http://creativecommons.org/ licenses/by-nc/4.0) which permits unrestricted non-commercial use, distribution, and reproduction in any medium, provided the original work is properly cited.

Copyright (c) The Korean Dermatological Association and The Korean Society for Investigative Dermatology 

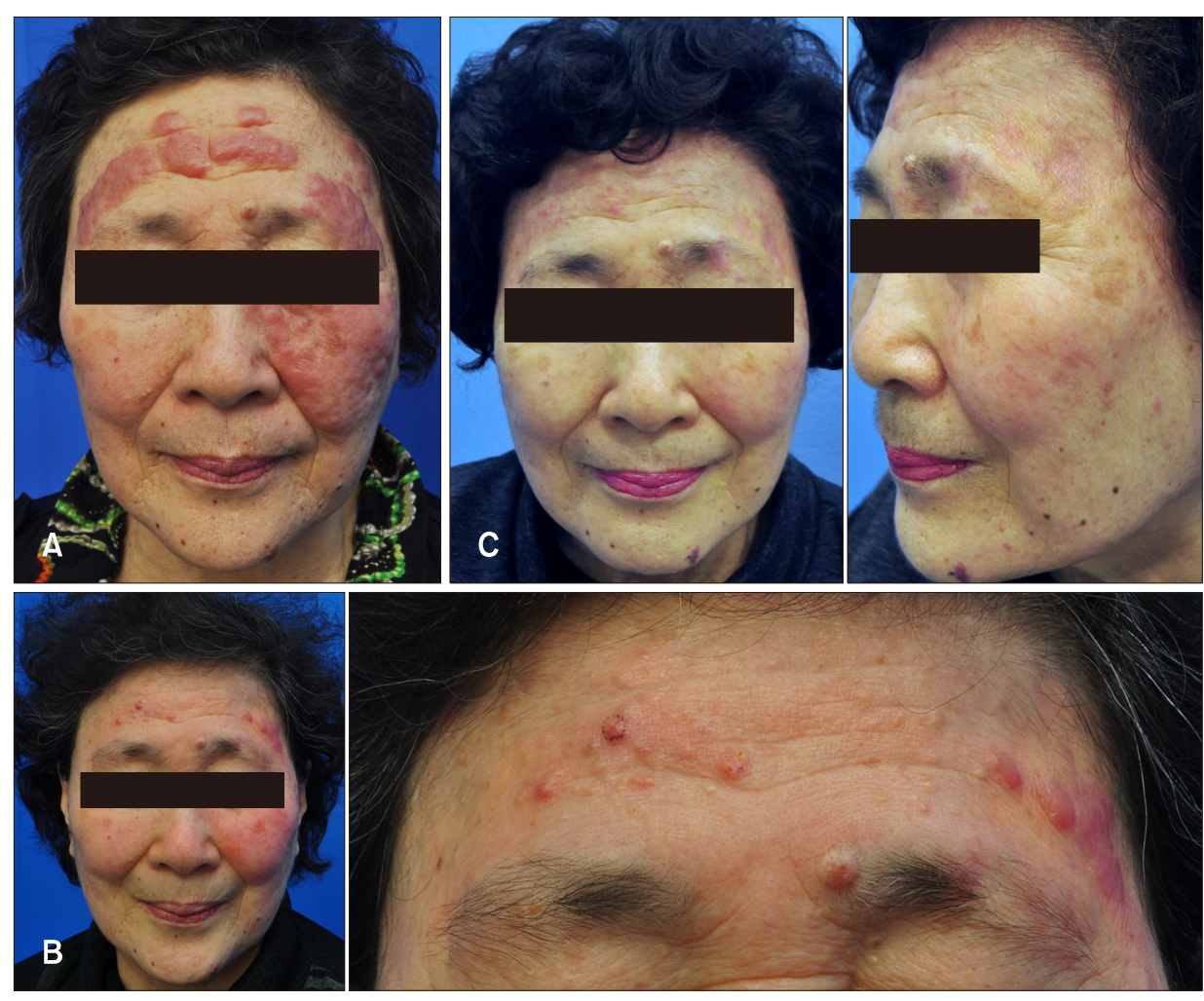

Fig. 1. Clinical characteristics. (A) Initial clinical presentation of patient. Multiple confluent erythematous plaques on the forehead and left side of her face; (B) Recurrence of erythematous plaques with multiple milia found within the lesions; (C) Significant improvement of the lesions after the extraction of milia. We received the patient's consent form about publishing all photographic materials.
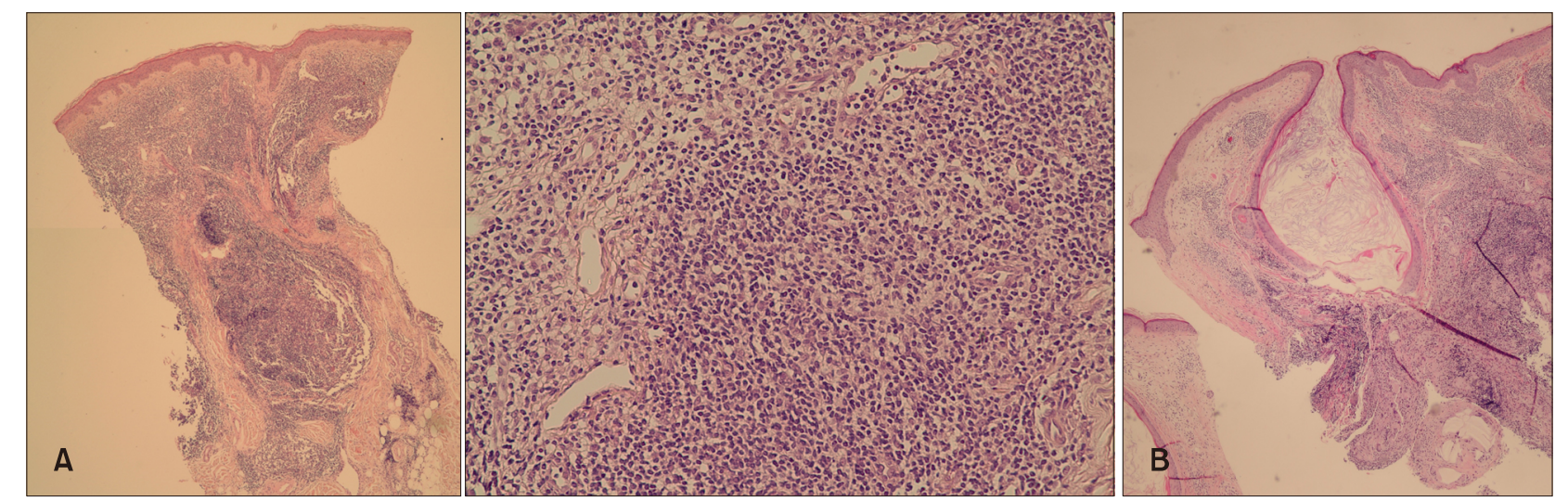

Fig. 2. Histological findings. (A) Histological examination shows lymphoid nodules consisting of dense mixed lymphocytic infiltrates $(\mathrm{H} \& \mathrm{E}$; left, $\times 40$ and right, $\times 200$ magnification). (B) Histologic examination showing dense lymphocytic infiltration visibly derived from rupture of milia ( $\mathrm{H} \&, \times 40$ magnification).

to the development of CPL.

To our knowledge, this is the first case report of CPL associated with milia although further studies are necessary. Spontaneous resolution of CPL may be induced by elimination of the underlying skin pathology which can be a persistent antigenic stimulus.

\section{SUPPLEMENTARY MATERIALS}

Supplementary data can be found via http://anndermatol. org/src/sm/ad-30-725-s001.pdf.

\section{CONFLICTS OF INTEREST}

The authors have nothing to disclose.

\section{REFERENCES}

1. Bergman R. Pseudolymphoma and cutaneous lymphoma: facts and controversies. Clin Dermatol 2010;28:568-574.

2. Cristaudo A, Forte G, Bocca B, Petrucci F, Muscardin L, Trento $E$, et al. Permanent tattoos: evidence of pseudolymphoma in three patients and metal composition of the 
dyes. Eur J Dermatol 2012;22:776-780.

3. Imafuku S, Ito K, Nakayama J. Cutaneous pseudolymphoma induced by adalimumab and reproduced by infliximab in a patient with arthropathic psoriasis. Br J Dermatol 2012;166: 675-678.

4. Nihal M, Mikkola D, Horvath N, Gilliam AC, Stevens SR, Spiro TP, et al. Cutaneous lymphoid hyperplasia: a lympho- proliferative continuum with lymphomatous potential. Hum Pathol 2003;34:617-622.

5. Burg G, Dummer R, Haeffner A, Kempf W, Kadin M. From inflammation to neoplasia: mycosis fungoides evolves from reactive inflammatory conditions (lymphoid infiltrates) transforming into neoplastic plaques and tumors. Arch Dermatol 2001;137:949-952.

\title{
CD13 Expression in Onychomatricoma: Association with Nail Matrix Onychodermis
}

\author{
Chan Seong Park, Ji-Hye Park, Dong-Youn Lee \\ Department of Dermatology, Samsung Medical Center, Sungkyunkwan University School of Medicine, Seoul, Korea
}

\section{Dear Editor:}

Onychomatricoma is a rare peculiar tumor of the nail unit. It was originally reported to be a benign tumor of the nail matrix as its name implies. However, the terminology onychomatricoma may be a misnomer. Histologically, it is a fibroepithelial tumor consisting of epithelial and mesenchymal components. Thus, the concept of epithelial onychogenic tumor with onychogenic mesenchyme was suggested ${ }^{1}$.

Recently, we found the presence of onychodermis of Dongyoun, a specialized mesenchymal cell population below the the nail matrix and proximal nail bed demonstrating CD10 expression ${ }^{2}$. Considering the components and location of onychomatricoma and its CD10 expression, we suggested the relation of onychomatricoma to onychodermis in the nail unit ${ }^{3}$. In addition, very recently, we demonstrated that stronger CD13 expression was found in the mesenchyme containing onychofibroblasts below the nail matrix compared to that below the nail bed, suggesting that CD13 may be a marker for onychofibroblasts within nail matrix onychodermis ${ }^{4}$.

In this study, to evaluate CD13 expression in onychomatricoma and further elucidate the association of onychomatricoma with onychodermis in the nail unit we performed CD 13 immunohistochemistry in three onychomatricoma cases (one case was diagnosed at Samsung Medical Center [Fig. 1]. The other two cases were kind gifts from Dr. Robert Baran and from Catholic University Hospital, Seoul, Korea). Immunohistochemical staining was performed using the monoclonal antibody to CD13 (1:50; clone 38C 12; Abcam, Cambridge, UK).

$\mathrm{H} \& \mathrm{E}$ staining showed a fibroepithelial tumor with characteristic digitations. The tumor was lined by nail matrix-like epithelium. The tumor cells consisted of spindle shaped nuclei. Immunohistochemically, CD13 was strongly expressed diffusely in dermal portion of onychomatricoma (Fig. 2). CD13 expression was very similar in thee onychomatricoma cases.

\footnotetext{
Received November 3, 2017, Revised December 11, 2017, Accepted for publication December 14, 2017
}

Corresponding author: Dong-Youn Lee, Department of Dermatology, Samsung Medical Center, Sungkyunkwan University School of Medicine, 81 Irwon-ro, Gangnam-gu, Seoul 06351, Korea. Tel: 82-2-3410-3543, Fax: 82-2-3410-3869, E-mail: dylee@skku.edu ORCID: https://orcid.org/0000-0003-0765-9812

This is an Open Access article distributed under the terms of the Creative Commons Attribution Non-Commercial License (http://creativecommons.org/ licenses/by-nc/4.0) which permits unrestricted non-commercial use, distribution, and reproduction in any medium, provided the original work is properly cited.

Copyright $($ The Korean Dermatological Association and The Korean Society for Investigative Dermatology 


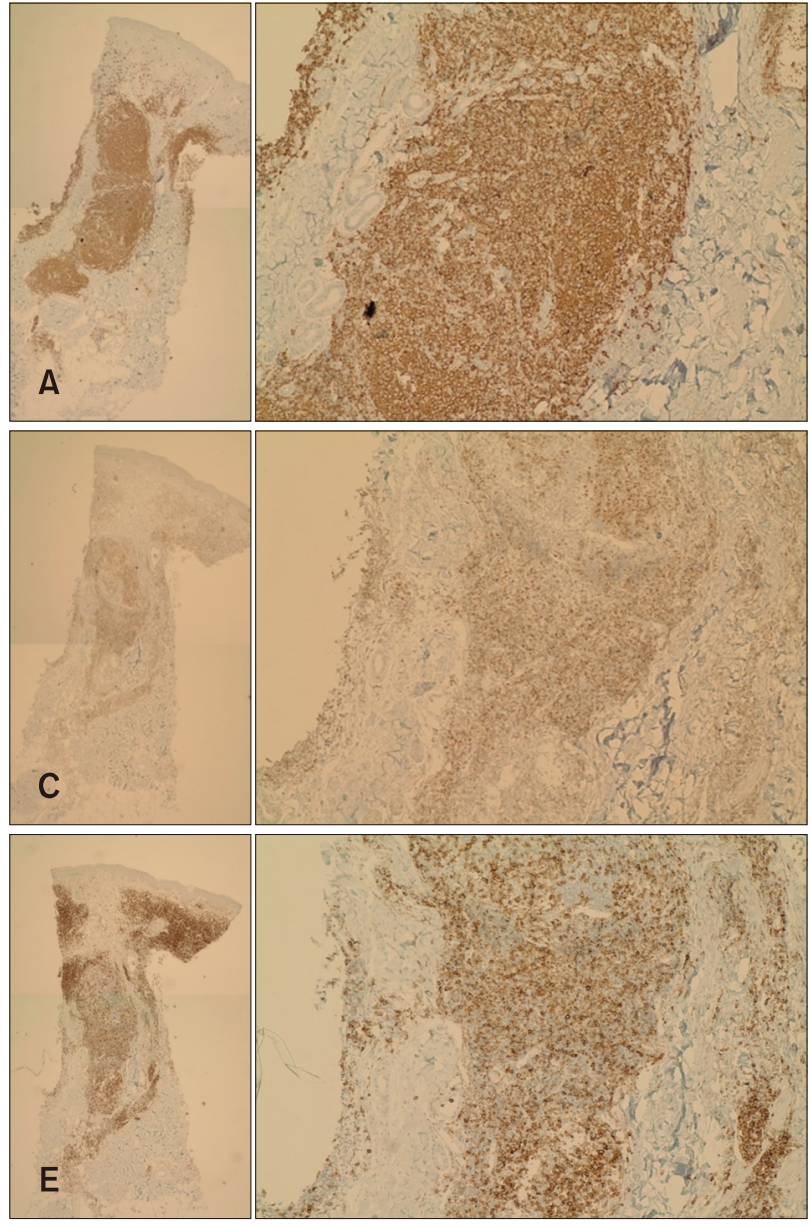

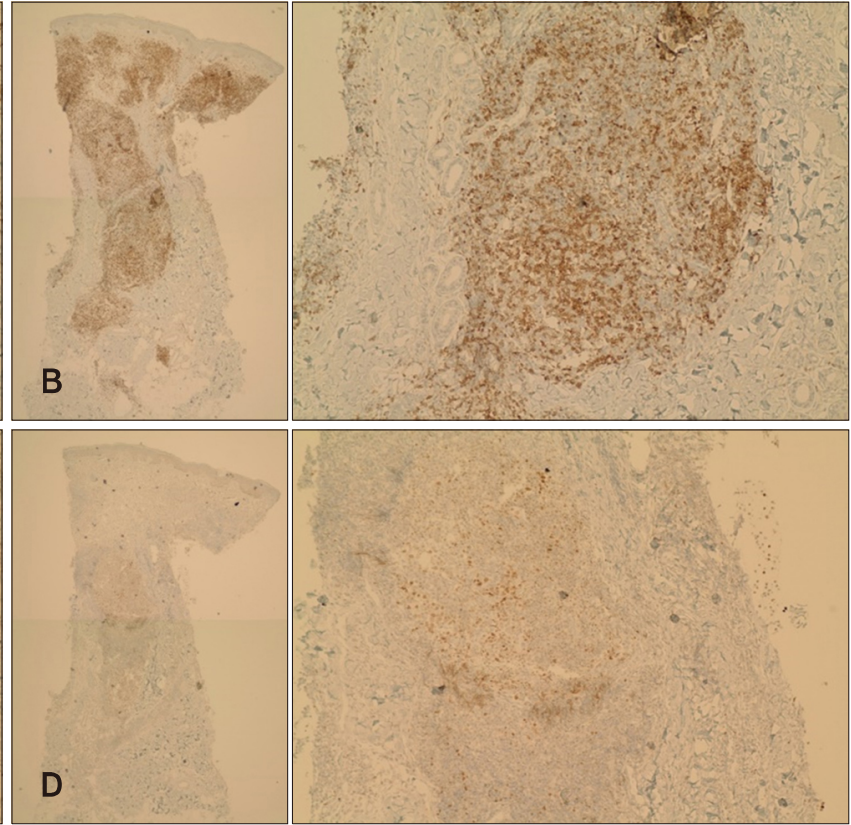

Supplementary Fig. 1. Immunohistochemistry. (A) Positive immunostaining for CD20 antigen denotes B lymphocytes; (B) Positive immunostaining for $\mathrm{CD} 3$ antigen denotes $\mathrm{T}$ lymphocytes; (C) Focally positive immunostaining for BCL2 antigen; (D) Focally positive immunostaining for BCL6 antigen; (E) Positive immunostaining for CD43 antigen denotes T lymphocytes (left, $\times 40$ and right, $\times 100$ magnification). 